\title{
Peristaltic Motion of Viscoelastic Fluid with Fractional Second Grade Model in Curved Channels
}

\author{
V. K. Narla, ${ }^{1}$ K. M. Prasad, ${ }^{1}$ and J. V. Ramanamurthy ${ }^{2}$ \\ ${ }^{1}$ Department of Mathematics, GITAM University, Hyderabad-502329, Andhra Pradesh, India \\ ${ }^{2}$ Department of Mathematics, National Institute of Technology, Warangal-506004, Andhra Pradesh, India
}

Correspondence should be addressed to V. K. Narla; vknarla@gmail.com

Received 11 October 2013; Accepted 27 November 2013

Academic Editors: H. Hu and Y. Zhu

Copyright (c) 2013 V. K. Narla et al. This is an open access article distributed under the Creative Commons Attribution License, which permits unrestricted use, distribution, and reproduction in any medium, provided the original work is properly cited.

Exact analytic solutions are obtained for the flow of a viscoelastic fluid with fractional second grade model by peristalsis through a curved channel. The flow has been investigated under the assumptions of long wavelength and low Reynolds number approximation. The streamlines for trapped bolus of Newtonian fluid are analyzed graphically. The fractional calculus approach is used to get analytic solutions of the problem. The influence of fractional parameter, material constant, amplitude, and curvature parameter on the pressure and friction force across one wavelength are discussed numerically with the help of graphs.

\section{Introduction}

Peristalsis is a mechanism of fluid transport through deformable vessels with the aid of a progressive contraction/expansion wave along the vessel. This mechanism appears to be a major mechanism for fluid transport in many physiological systems. It appears in the gastrointestine tract, urine transport from kidney to bladder, bile from the gall bladder into the duodenum, the movement of spermatoza in the ducts efferentes of the male reproductive tract, transport of lymph in the lymphatic vessels, and in the vasomotion of small blood vessels such as arterioles, venules, and capillaries. Peristaltic fluid transport is being increasingly used by modern technology in cases where it is necessary to avoid contact between the pumped medium and the mechanical parts of the pump.

A mathematical model to understand fluid mechanics of this phenomenon has been developed using lubrication theory, provided that the fluid inertia effects are negligible and the flow is of the low Reynolds number. The flow of Newtonian and non-Newtonian fluids was described by many researchers in straight vessels (Shapiro et al. [1], Jaffrin and Shapiro [2], Jaffrin [3], Pozrikidis [4], Vajravelu et al. [5], and Li and Brasseur [6]). In recent years it has turned out that the mathematical models in areas like viscoelasticity and electrochemistry as well as in many fields of science and engineering including fluid flow, rheology, diffusive transport, electrical networks, electromagnetic theory, and probability can be formulated very successfully by fractional calculus. In particular, it has been found to be quite flexible in describing viscoelastic behavior of fluids. The starting point of the fractional derivative model of non-Newtonian fluids is usually a classical differential equation which is modified by replacing the time derivative of an integer order by the so-called Riemann-Liouville fractional operator. The fractional derivative models have been used in various situations to analyze diverse rheological problems. The fractional second grade model is one among these fractional models. Wenchang et al. [7] have investigated the unsteady flow of viscoelastic fluid with fractional Maxwell model between two parallel plates. Qi and Jin [8] have discussed unsteady flows between coaxial cylinders, while Qi and Xu [9] have studied the flow properties. Hayat et al. [10] have constructed periodic unidirectional flows of a viscoelastic fluid with the fractional Maxwell model and solutions are solved by Fourier transform. Khan et al. [11] have discussed the decay of potential vertex for viscoelastic fluid with fractional Maxwell model and analytical solutions are obtained by Hankel transform and discrete Laplace transform. Recently, Tripathi et al. have studied the peristaltic flow of viscoelastic fluid with fractional models [12-19]. 
In all these previous studies authors have carried out the analysis in straight channels or tubes. In fact, the shape of most physiological ducts are curved. The corresponding study of the peristaltic flow in curved channel was carried out by Sato et al. [20]. Ali et al. [21] discussed the peristaltic motion in a curved channel using wave frame. Later, Ali et al. [22] extended the flow analysis by considering heat transfer effects. Hayat et al. [23] have examined the peristaltic flow of viscous fluid in a curved channel with complaint walls. NonNewtonian fluid flow induced by peristaltic waves in a curved channel has been investigated by Ali et al. [24]. Hayat et al. [25] have extended the problem to investigate the effect of an induced magnetic field on peristaltic flow of non-Newtonian fluid in a curved channel. Very recently, Ramanamurthy et al. [26] have investigated unsteady effects of peristaltic transport in curved channels.

The objective of this paper is to investigate the effects of fractional parameters of second grade fluid on peristaltic flow through a curved channel. In order to study these effects we employed long wave length and the low Reynolds number approximation. The fractional calculus approach is used to obtain analytical solution of the problem. The obtained expressions are utilized to discuss the influences of various physical parameters.

\section{Preliminaries}

Here we present the necessary definitions from fractional calculus theory, which are useful in subsequent sections. These definitions can be found in the recent literature [7, 27, 28].

Definition 1. The Riemann-Liouville fractional integral operator of order $\alpha>0$ of a function $f(x):(0, \infty) \rightarrow \mathbb{R}$ is given by

$$
J^{\alpha} f(x)=\frac{1}{\Gamma(\alpha)} \int_{0}^{x}(x-\xi)^{\alpha-1} f(\xi) d \xi, \quad \alpha>0 x>0 .
$$

Definition 2. The fractional derivative of order $\alpha>0$ of a continuous function $f(x):(0, \infty) \rightarrow \mathbb{R}$ is given by

$$
\begin{array}{r}
D^{\alpha} f(x)=\frac{1}{\Gamma(m-\alpha)}\left(\frac{d}{d x}\right)^{m} \int_{0}^{x}(x-\xi)^{m-\alpha-1} f(\xi) d \xi, \\
\text { for } m-1<\alpha \leq m, m \in \mathbb{N}, x>0, f \in \mathbb{C}_{-1}^{m},
\end{array}
$$

where $m=[\alpha]+1$, provided that the right-hand side is pointwise defined on $(0, \infty)$.

Remark 3. For example $f(x)=x^{\beta}$, we quote for $\beta>-1$, in (2) one can get

$$
D^{\alpha} x^{\beta}=\frac{\Gamma(\beta+1)}{\Gamma(\beta-\alpha+1)} x^{\beta-\alpha},
$$

giving in particular $D^{\alpha} x^{\beta-n}=0, n=1,2, \ldots, N$, where $N$ is the smallest integer greater than or equal to $\alpha$.

\section{Mathematical Model}

We consider the flow of a fluid modeled as a fractional second grade model given by

$$
\tau=\mu\left(1+\lambda_{1}^{\alpha} \frac{\partial^{\alpha}}{\partial t^{\alpha}}\right) \dot{\gamma}
$$

where $\lambda_{1}, \alpha, t, \tau, \mu$, and $\dot{\gamma}$ are the relaxation time, fractional parameter, time, shear stress, viscosity, and rate of shear strain.

We consider the flow of an incompressible, viscoelastic fluid with fractional second grade model in a curved channel induced by two infinite trains of sinusoidal waves that are propagated along the flexible walls of the channel. When undeformed, the walls are separated by a distance $2 a$; the constant radius of curvature at the channel centreline is $R$. We choose curvilinear coordinate system $(x, r)$ in such a way that $x$-axis lies along the center line of the curved channel and $r$-axis is normal to it and is measured from central line. The scale factors are $h_{1}=(r+R) / R, h_{2}=1$, and $h_{3}=1$. There is no component in $z$ direction as shown in Figure 1.

The sinusoidal waves propagating along the channel walls are described as

$$
\begin{gathered}
r=h(x, t)=a+b \cos \left[2 \pi\left(\frac{x}{\lambda}-\frac{t}{T}\right)\right](\text { Upper wall), } \\
r=-h(x, t)=-a-b \cos \left[2 \pi\left(\frac{x}{\lambda}-\frac{t}{T}\right)\right] \text { (Lower wall). }
\end{gathered}
$$

Here, $x$ is the axial distance, $a$ the radius of the stationary curved channel, $\phi$ the wave amplitude, $\lambda$ the wave length, $T$ the wave period, and $h$ the radial displacement of the wave from the centerline. The wavelength is large compared with the channel's width $(a / \lambda \ll 1)$.

The governing equations for an ordinary second grade fluid through curved channel with the velocity vector $\bar{V}=$ $u \widehat{e}_{x}+v \widehat{e}_{r}$ are given by

$$
R \frac{\partial u}{\partial x}+\frac{\partial}{\partial r}\{(r+R) v\}=0
$$

$$
\begin{aligned}
\frac{\partial u}{\partial t}+ & (\bar{V} \cdot \nabla) u-\frac{u v}{r+R} \\
= & -\frac{R}{\rho(r+R)} \frac{\partial p}{\partial x}+v\left(1+\lambda_{1} \frac{\partial}{\partial t}\right) \\
& \times\left[\nabla^{2} u-\frac{u}{(r+R)^{2}}+\frac{2 R}{(r+R)^{2}} \frac{\partial v}{\partial x}\right],
\end{aligned}
$$




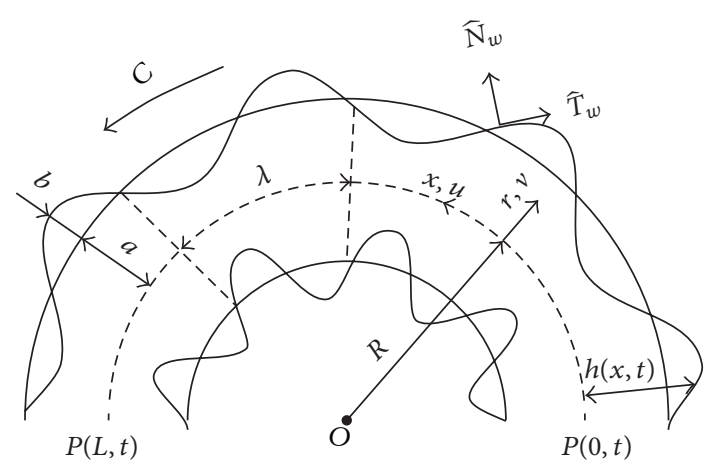

FIgURE 1: Peristaltic wave in curved channel.

$$
\begin{aligned}
\frac{\partial v}{\partial t}+ & (\bar{V} \cdot \nabla) v-\frac{u^{2}}{r+R} \\
= & -\frac{1}{\rho} \frac{\partial p}{\partial r}+v\left(1+\lambda_{1} \frac{\partial}{\partial t}\right) \\
& \times\left[\nabla^{2} v-\frac{v}{(r+R)^{2}}-\frac{2 R}{(r+R)^{2}} \frac{\partial u}{\partial x}\right],
\end{aligned}
$$

where

$$
\begin{gathered}
(\bar{V} \cdot \nabla)=\frac{R u}{(r+R)} \frac{\partial}{\partial x}+v \frac{\partial}{\partial r} \\
\nabla^{2}=\left(\frac{R}{r+R}\right)^{2} \frac{\partial^{2}}{\partial x^{2}}+\frac{1}{r+R} \frac{\partial}{\partial r}+\frac{\partial^{2}}{\partial r^{2}} .
\end{gathered}
$$

In the above equations $p$ is pressure, $\rho$ is fluid density, $v$ is the kinematic viscosity, and $u$ and $v$ are the velocity components in radial $r$ and axial $x$ directions, respectively.

The following dimensionless variables and parameter are introduced:

$$
\begin{gathered}
x^{\prime}=\frac{x}{\lambda}, \quad r^{\prime}=\frac{r}{a}, \quad u^{\prime}=\frac{u}{c}, \\
v^{\prime}=\frac{v}{\delta c}, \quad h^{\prime}=\frac{h}{a}, \quad \phi=\frac{b}{a}, \quad \kappa=\frac{R}{a}, \\
p^{\prime}=\frac{a^{2} p}{\mu c \lambda}, \quad \operatorname{Re}=\frac{c a \delta}{v}, \quad \psi^{\prime}=\frac{\psi}{a c}, \\
Q=\frac{Q}{a c}, \quad t^{\prime}=\frac{t}{T},
\end{gathered}
$$

where $\operatorname{Re}$ is Reynolds number, $Q$ is volume flow rate, $c=\lambda / T$ is the velocity of the wave, $\delta=a / \lambda$ defines wave number, $\phi$ is the amplitude ratio, and $\kappa$ is the curvature parameter.

The stream function $\psi$ can be defined by using dimensionless variables and parameters in (10), which satisfies continuity equation (6) as

$$
u=-\frac{\partial \psi}{\partial r}, \quad v=\frac{\kappa}{r+\kappa} \frac{\partial \psi}{\partial x} .
$$

The dimensionless equations are obtained (neglecting the primes for clarity) by applying long wavelength and negligible Reynolds number approximation using (4), (7), and (8) as

$$
\begin{gathered}
\frac{\partial p}{\partial x}=\left(1+\lambda_{1}^{\alpha} \frac{\partial^{\alpha}}{\partial t^{\alpha}}\right)\left(\frac{1}{\kappa} \frac{\partial}{\partial r}\left\{(r+\kappa) \frac{\partial^{2} \psi}{\partial^{2} r}\right\}-\frac{1}{\kappa(r+\kappa)} \frac{\partial \psi}{\partial r}\right), \\
\frac{\partial p}{\partial r}=0 .
\end{gathered}
$$

The dimensionless boundary conditions are given by

$$
\begin{aligned}
& \psi=-\frac{Q(x, t)}{2}, \quad \frac{\partial \psi}{\partial r}=0, \quad \text { at } r=h(x, t), \\
& \psi=\frac{Q(x, t)}{2}, \quad \frac{\partial \psi}{\partial r}=0, \quad \text { at } r=-h(x, t),
\end{aligned}
$$

where $Q(x, t)$ is volume flow rate and is defined as $Q(x, t)=$ $\int_{-h}^{h} u(x, r, t) d r$.

\section{Solution of the Problem}

The transformations between the wave frame and the laboratory frame, in dimensionless form, are defined as

$$
\begin{array}{ll}
X=x-c t, \quad Y=r, & U=u-1, \\
V=v, \quad q=Q-2 h, & \Psi=\psi-r,
\end{array}
$$

where the parameters on the left side are in the wave frame and thoseon the right side are in the laboratory frame. The solutions of (12) and (13) with the boundary conditions equation (14) in wave frame of reference are obtained as

$$
\begin{gathered}
\Psi=C_{1}+C_{2} \log (Y+\kappa)+C_{3}(Y+k)^{2} \\
+C_{4}(Y+\kappa)^{2} \log (Y+\kappa)+(Y+\kappa), \\
C_{1}=-\frac{1}{2}[2 H+q+2 \kappa \\
+\left((H+q)^{2}(2 H+q)\right. \\
\quad \times\left(2 H \kappa+(H-\kappa)^{2}\right. \\
\quad \times(1-2 \log (H+\kappa)) \\
\quad \times \log ((H+\kappa) /(H-\kappa))))
\end{gathered}
$$




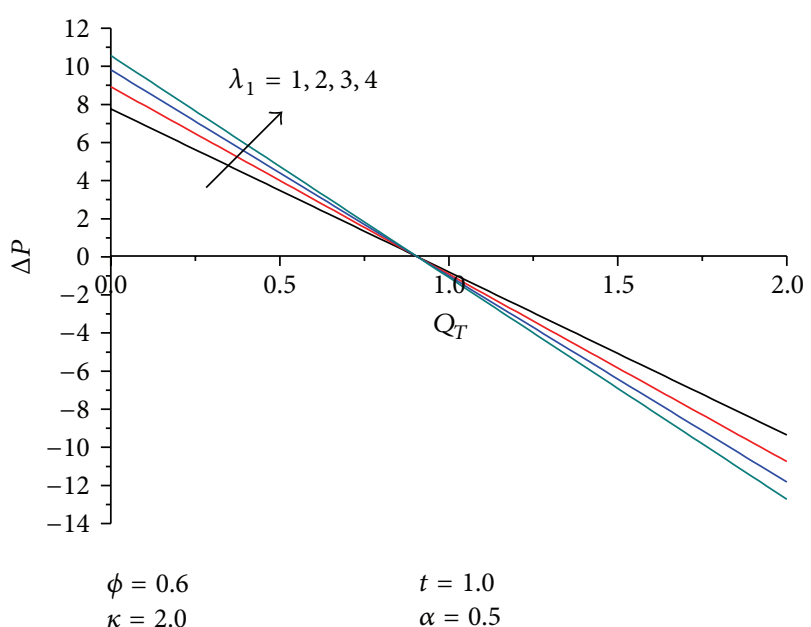

(a)

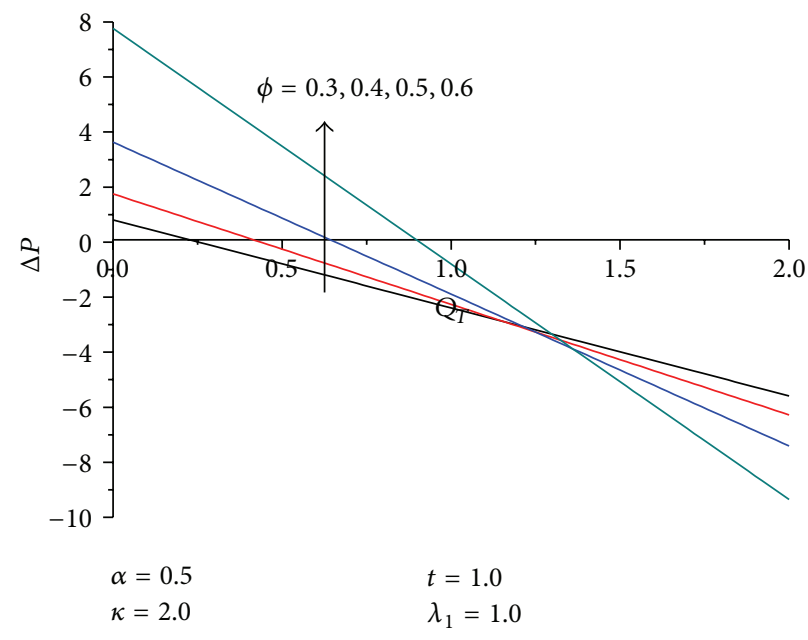

(c)

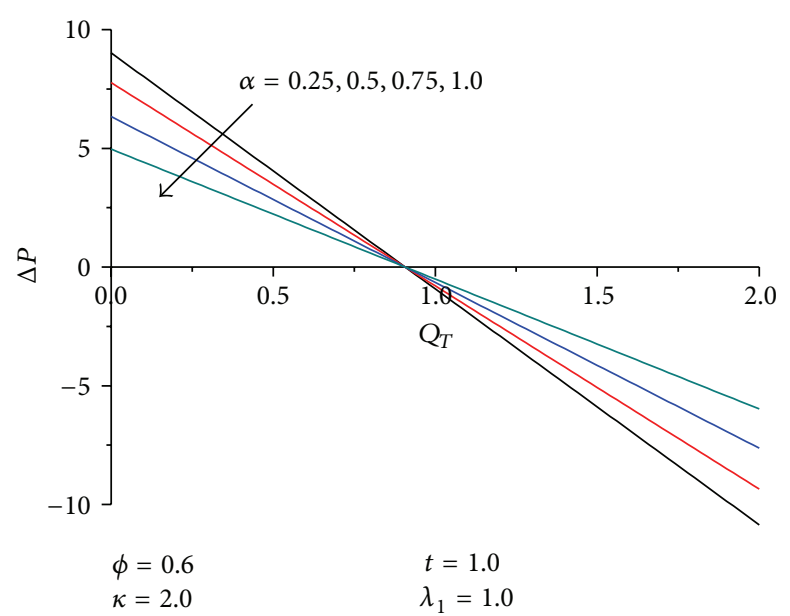

(b)

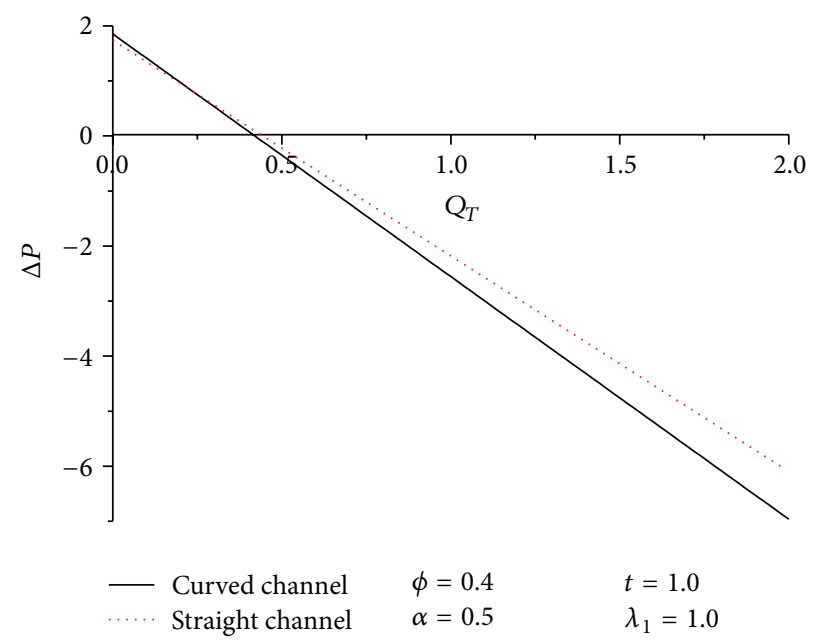

(d)

FIGURE 2: Pressure difference across one wavelength against time-averaged flow rate.

$$
\begin{gathered}
\times\left(-4 H^{2} \kappa^{2}+\left(H^{2}-\kappa^{2}\right)^{2}\right. \\
\left.\left.\times(\log ((\kappa-H) /(\kappa+H)))^{2}\right)^{-1}\right], \\
C_{2}=\frac{(2 H+q)\left(H^{2}-\kappa^{2}\right)^{2} \log ((\kappa-H) /(\kappa+H))}{-4 H^{2} \kappa^{2}+\left(H^{2}-\kappa^{2}\right)^{2}(\log ((\kappa-H) /(\kappa+H)))^{2}}, \\
C_{3}=-((2 H+q) \\
\times\left[-2 H \kappa+(H-\kappa)^{2} \log (\kappa-H)\right. \\
\left.\left.-(H+\kappa)^{2} \log (H+\kappa)\right]\right) \\
\times\left(2 \left[-4 H^{2} \kappa^{2}+\left(H^{2}-\kappa^{2}\right)^{2}\right.\right. \\
\left.\left.\times(\log ((\kappa-H) /(\kappa+H)))^{2}\right]\right)^{-1}, \\
2(2 H+q) H \kappa
\end{gathered}
$$

where the channel wall takes the form $H(X)=1+\phi \cos (2 \pi X)$. The axial pressure gradient in terms of time-averaged flow can be obtained by substituting (16) in (12) as

$$
\begin{aligned}
\frac{\partial p}{\partial X}(X, t) & \\
= & \left(1+\lambda_{1}^{\alpha} \frac{\partial^{\alpha}}{\partial t^{\alpha}}\right) \\
& \times \frac{8(2 H+q) H}{-4 H^{2} \kappa^{2}+\left(H^{2}-\kappa^{2}\right)^{2}(\log ((\kappa-H) /(\kappa+H)))^{2}} .
\end{aligned}
$$

Using Definition 2 in (17), we get the pressure gradient

$$
\begin{aligned}
\frac{\partial p}{\partial X}(X, t) & \frac{8(2 H+q) H}{-4 H^{2} \kappa^{2}+\left(H^{2}-\kappa^{2}\right)^{2}(\log ((\kappa-H) /(\kappa+H)))^{2}} \\
& \times\left(1+\lambda_{1}^{\alpha} \frac{t^{-\alpha}}{\Gamma(1-\alpha)}\right) .
\end{aligned}
$$




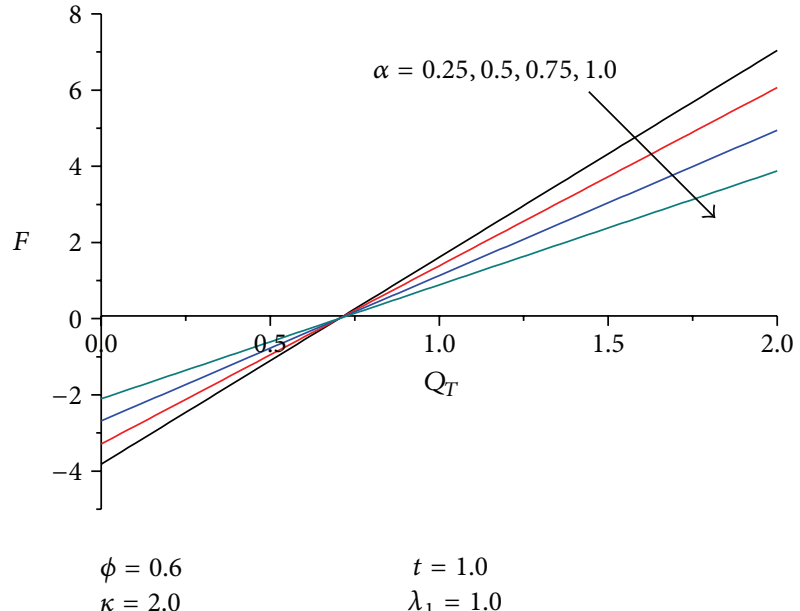

(a)

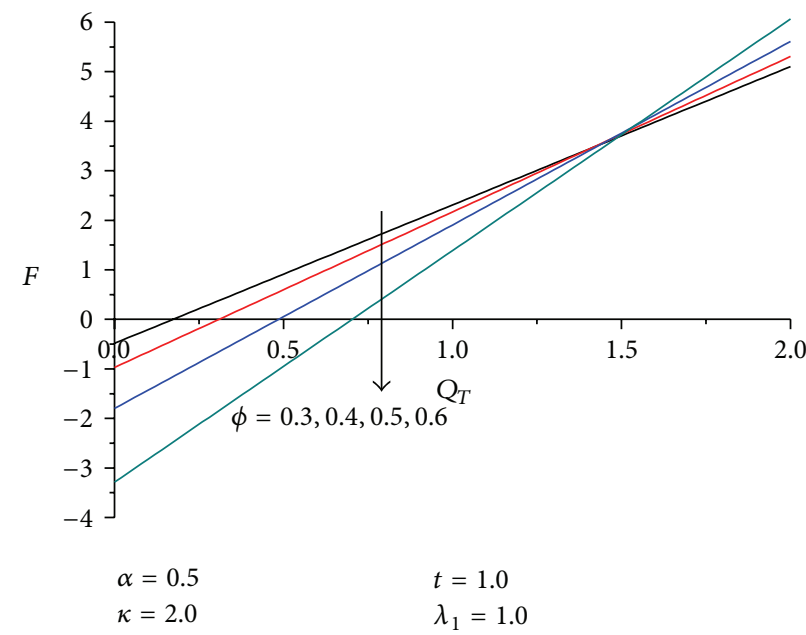

(c)

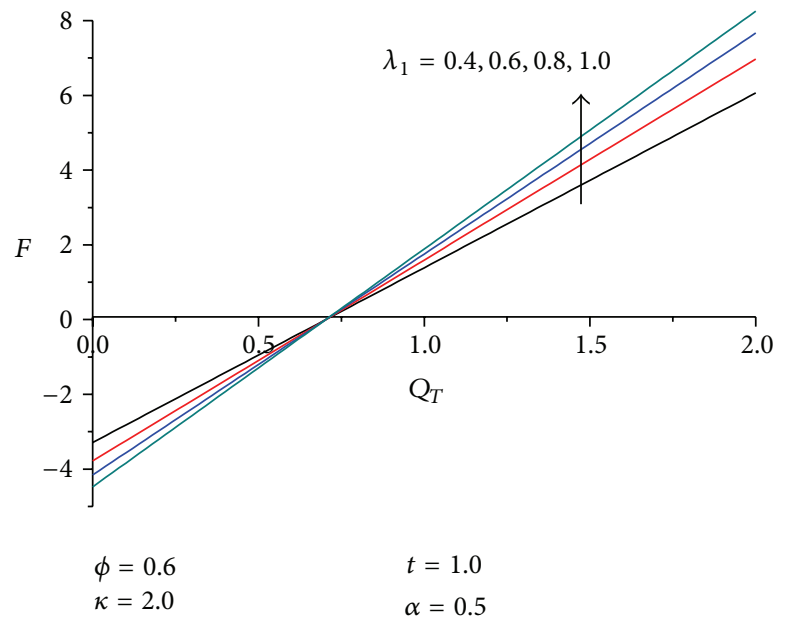

(b)

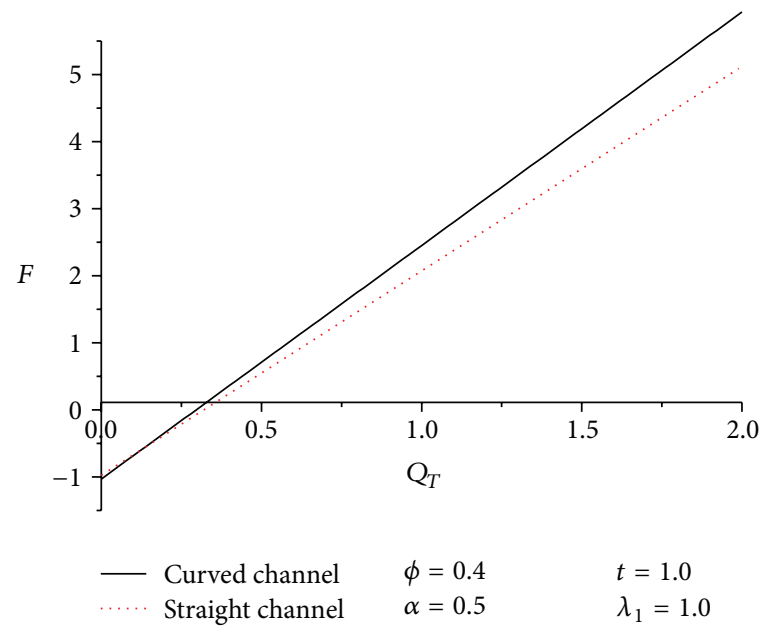

(d)

FIGURE 3: Friction force across one wavelength against time-averaged flow rate.

The pressure difference $\Delta p$ and friction force $F$ across one wavelength are given by

$$
\begin{aligned}
& \Delta p=\int_{0}^{1} \frac{\partial p}{\partial x} d x \\
& F=\int_{0}^{1}-h \frac{\partial p}{\partial x} d x
\end{aligned}
$$

\section{Results and Discussion}

In this paper, we analyze the peristaltic motion of fractional second grade fluid through curved channel. Exact solution for stream function is obtained. Based on this exact solution, we discuss the effects of various pertinent parameters such as fractional parameter $(\alpha)$, material constant $\left(\lambda_{1}\right)$, occlusion parameter $(\phi)$, and curvature parameter $(\kappa)$ on pressure difference across one wavelength $(\Delta p)$ and friction force across the one wavelength $(F)$.

The pressure difference across one wavelength is plotted against averaged flow rate in Figures 2(a)-2(d) for different values of the fractional parameters $(\alpha=0.25,0.5,0.75,1.0)$, channel curvature, relaxation time $\left(\lambda_{1}=0.4,0.6,0.8,1.0\right)$, and amplitude $(\phi=0.3,0.4,0.5,0.6)$, respectively. One may observe from these figures that the pressure-flow relation is linear. The present study examined three regions of peristaltic motion with positive pumping $\left(Q_{T}>0\right)$, in the entire pumping region $(\Delta p>0)$, in the free pumping region $(\Delta p=0)$, and in the copumping region $(\Delta p<0)$. It may be noted from Figure 2(a) that the volumetric flow rate can be gradually increased in the pumping region and gradually decreased in the copumping region by increasing the value of relaxation time $\lambda_{1}$. Figure 2(b) shows that in the entire pumping region the volumetric flow rate decreases with the increase in fractional parameter $\alpha$, whereas in the copumping region, a reverse trend is noticed. It is observed from Figure 2(c) that, in the range of values of pressure gradient $\Delta p>-3.2$, the volumetric flow rate increases with the increase in the amplitude ratio $\phi$. However, the trend reverses as soon as the pressure gradient drops below -3.2 . Figure 2(d) reveals that the flow rate slightly increases with increasing channel curvature in the pumping region as well as copumping region. 


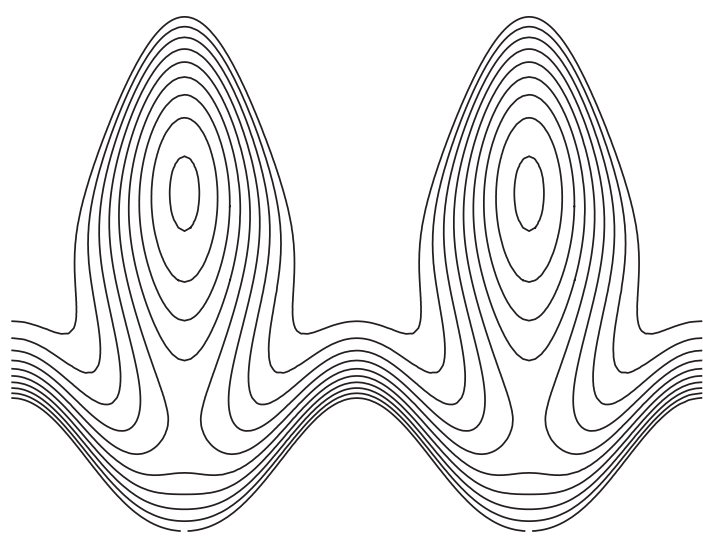

(a) $\kappa=3$

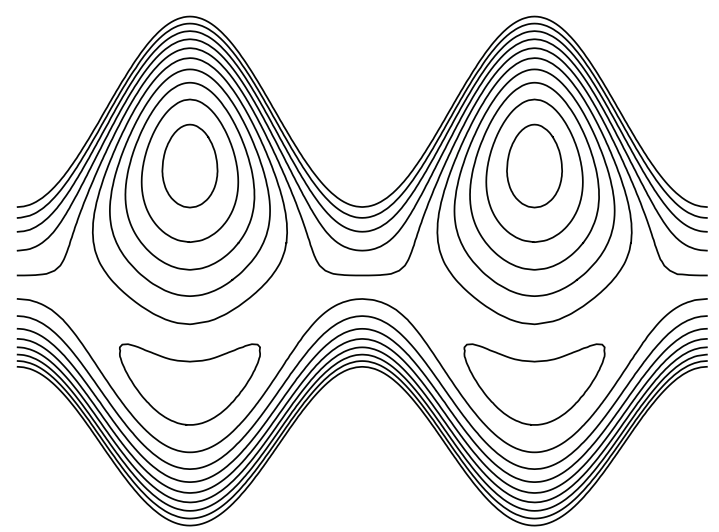

(c) $\kappa=12$

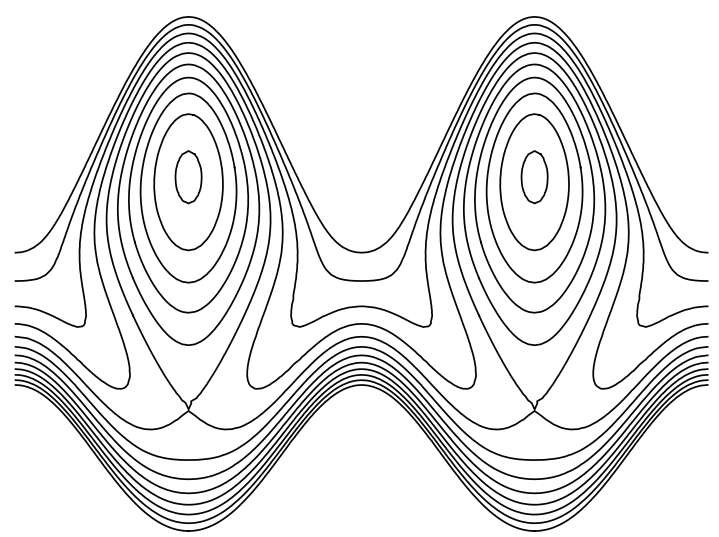

(b) $\kappa=5$

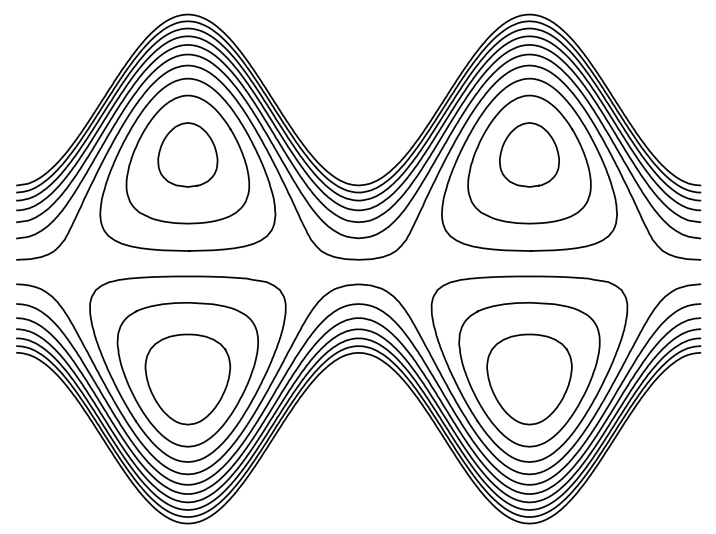

(d) $\kappa \rightarrow \infty$

FIGURE 4: Streamline patterns at different channel curvatures.

Figures 3(a)-3(d) show the variations of friction force $F$ with the averaged flow rate $Q_{T}$ under the influence of all parameters $\alpha, \kappa, \lambda_{1}$, and $\phi$. It is observed from the figures that friction forces have opposite behavior in comparison with pressure.

Trapping is an important phenomenon described as a bolus of fluid that moves as a whole with the wave [1]. Trapping may be observed in curved channel by plotting the streamlines in a wave frame [4]. The streamline patterns in the wave frame for Newtonian fluid with $Q_{T}=1.5$ and $\phi=$ 0.5 for different values of curvature parameter $\kappa$ are shown in Figure 4. It is observed that for small values of $\kappa$ only one trapped bolus is formed. There exists two asymmetric boluses as one moving from curved channel to straight channel. It is also observed that the bolus near the upper wall increases but the bolus near lower wall disappears eventually as the channel curvature is increased. However, the result agrees almost well for straight channel $(\kappa \rightarrow \infty)$ as the bolus splits with almost equal magnitudes $[1,4]$.

\section{Concluding Remarks}

This paper analyzes a viscoelastic fluid flow with fractional second grade model for peristaltic motion in two-dimensional curved channels. The effects of fractional parameter, curvature of the channel, and viscoelastic behaviors on peristaltic motion in curved channel are studied. We obtained the analytical solution for stream function at low inertial effect when the wavelength is moderately large compared to the channel width. An approximate analytical solution for pressure gradient is obtained by fractional calculus theory. The following conclusions can be summarized.

(1) The relation between pressure and flow is found to be linear.

(2) The pressure-flow function decreases with increasing values of fractional parameter $\alpha$, curvature parameter $\kappa$, and amplitude ratio $\phi$, and it increases with increasing values of relaxation time $\lambda_{1}$.

(3) The variations of friction force against flow rate show opposite behavior to that of pressure.

(4) The streamlines in wave frame contain two asymmetrical parts: the bolus near the outer wall grows big and the bolus at inner wall diminishes as curvature of the channel increases.

\section{References}

[1] A. H. Shapiro, M. Y. Jaffrin, and S. L. Weinberg, "Peristaltic pumping with long wave length at Low Reynolds Number," Journal of Fluid Mechanics, vol. 37, no. 4, pp. 799-825, 1969. 
[2] M. Y. Jaffrin and A. H. Shapiro, "Peristaltic pumping," Annual Review of Fluid Mechanics, vol. 3, pp. 13-37, 1971.

[3] M. Y. Jaffrin, "Inertia and streamline curvature effects on peristaltic pumping," International Journal of Engineering Science, vol. 11, no. 6, pp. 681-699, 1973.

[4] C. Pozrikidis, "A study of peristaltic flow," Journal of Fluid Mechanics, vol. 180, pp. 515-527, 1987.

[5] K. Vajravelu, G. Radhakrishnamacharya, and V. Radhakrishnamurty, "Peristaltic flow and heat transfer in a vertical porous annulus, with long wave approximation," International Journal of Non-Linear Mechanics, vol. 42, no. 5, pp. 754-759, 2007.

[6] M. Li and J. G. Brasseur, "Non-steady peristaltic transport in finite-length tubes," Journal of Fluid Mechanics, vol. 248, pp. 129-151, 1993.

[7] T. Wenchang, P. Wenxiao, and X. Mingyu, "A note on unsteady flows of a viscoelastic fluid with the fractional Maxwell model between two parallel plates," International Journal of Non-Linear Mechanics, vol. 38, no. 5, pp. 645-650, 2003.

[8] H. Qi and H. Jin, "Unsteady rotating flows of a viscoelastic fluid with the fractional Maxwell model between coaxial cylinders," Acta Mechanica Sinica, vol. 22, no. 4, pp. 301-305, 2006.

[9] H. Qi and M. Xu, "Unsteady flow of viscoelastic fluid with fractional Maxwell model in a channel," Mechanics Research Communications, vol. 34, no. 2, pp. 210-212, 2007.

[10] T. Hayat, S. Nadeem, and S. Asghar, "Periodic unidirectional flows of a viscoelastic fluid with the fractional Maxwell model," Applied Mathematics and Computation, vol. 151, no. 1, pp. 153161, 2004.

[11] M. Khan, S. Hyder Ali, C. Fetecau, and H. Qi, "Decay of potential vortex for a viscoelastic fluid with fractional Maxwell model," Applied Mathematical Modelling, vol. 33, no. 5, pp. 2526-2533, 2009.

[12] D. Tripathi, S. K. Pandey, and S. K. Das, "Peristaltic flow of viscoelastic fluid with fractional Maxwell model through a channel," Applied Mathematics and Computation, vol. 215, no. 10, pp. 3645-3654, 2010.

[13] D. Tripathi, S. K. Pandey, and S. K. Das, "Peristaltic transport of a generalized Burgers' fluid: application to the movement of chyme in small intestine," Acta Astronautica, vol. 69, no. 1-2, pp. 30-38, 2011.

[14] D. Tripathi, "Peristaltic transport of a viscoelastic fluid in a channel," Acta Astronautica, vol. 68, no. 7-8, pp. 1379-1385, 2011.

[15] D. Tripathi, "Numerical study on peristaltic flow of generalized burgers' fluids in uniform tubes in the presence of an endoscope," International Journal for Numerical Methods in Biomedical Engineering, vol. 27, no. 11, pp. 1812-1828, 2011.

[16] D. Tripathi, "Peristaltic flow of a fractional second grade fluid through a cylindrical tube," Thermal Science, vol. 15, pp. 51675173, 2011.

[17] D. Tripathi, "Numerical and analytical simulation of peristaltic flows of generalized Oldroyd-B fluids," International Journal for Numerical Methods in Fluids, vol. 67, no. 12, pp. 1932-1943, 2011.

[18] D. Tripathi, "A mathematical model for the peristaltic flow of chyme movement in small intestine," Mathematical Biosciences, vol. 233, no. 2, pp. 90-97, 2011.

[19] D. Tripathi, "Peristaltic transport of fractional Maxwell fluids in uniform tubes: applications in endoscopy," Computers and Mathematics with Applications, vol. 62, no. 3, pp. 1116-1126, 2011.

[20] H. Sato, T. Kawai, T. Fujita, and M. Okabe, "Two-dimensional peristaltic flow in curved channels," Transactions of the Japan Society of Mechanical Engineers B, vol. 66, no. 643, pp. 679-685, 2000.
[21] N. Ali, M. Sajid, and T. Hayat, "Long wavelength flow analysis in a curved channel," Zeitschrift fur Naturforschung A, vol. 65, no. 3, pp. 191-196, 2010.

[22] N. Ali, M. Sajid, T. Javed, and Z. Abbas, "Heat transfer analysis of peristaltic flow in a curved channel," International Journal of Heat and Mass Transfer, vol. 53, no. 15-16, pp. 3319-3325, 2010.

[23] T. Hayat, M. Javed, and A. A. Hendi, "Peristaltic transport of viscous fluid in a curved channel with compliant walls," International Journal of Heat and Mass Transfer, vol. 54, no. 7-8, pp. 1615-1621, 2011.

[24] N. Ali, M. Sajid, Z. Abbas, and T. Javed, "Non-Newtonian fluid flow induced by peristaltic waves in a curved channel," European Journal of Mechanics B, vol. 29, no. 5, pp. 387-394, 2010.

[25] T. Hayat, S. Noreen, and A. Alsaedi, "Effect of an induced magnetic field on peristaltic flow of non-Newtonian fluid in a curved channel," Journal of Mechanics in Medicine and Biology, vol. 12, Article ID 125005, 26 pages, 2012.

[26] J. V. Ramanamurthy, K. M. Prasad, and V. K. Narla, "Unsteady peristaltic transport in curved channels," Physics of Fluids, vol. 25, Article ID 091903, 20 pages, 2013.

[27] G. Jumarie, "Table of some basic fractional calculus formulae derived from a modified Riemann-Liouville derivative for nondifferentiable functions," Applied Mathematics Letters, vol. 22, no. 3, pp. 378-385, 2009.

[28] C. Friedrich, "Relaxation and retardation functions of the Maxwell model with fractional derivatives," Rheologica Acta, vol. 30, no. 2, pp. 151-158, 1991. 

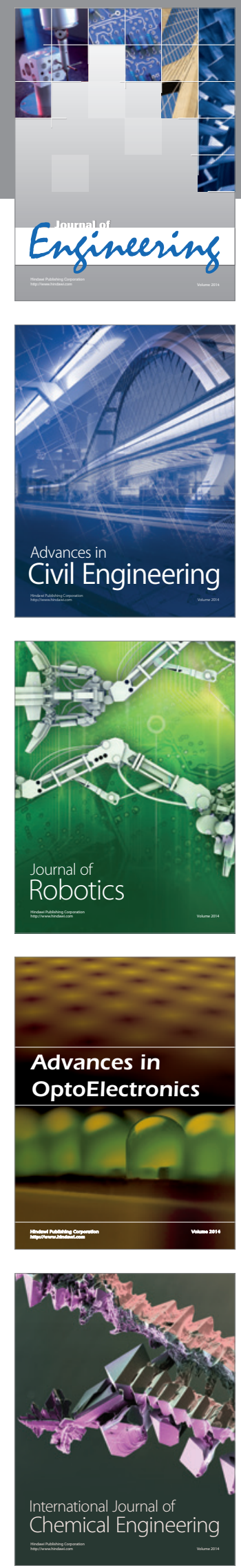

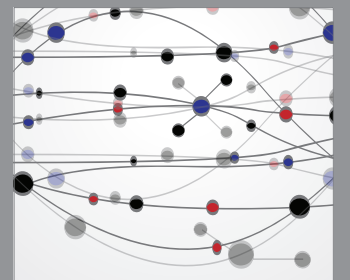

The Scientific World Journal
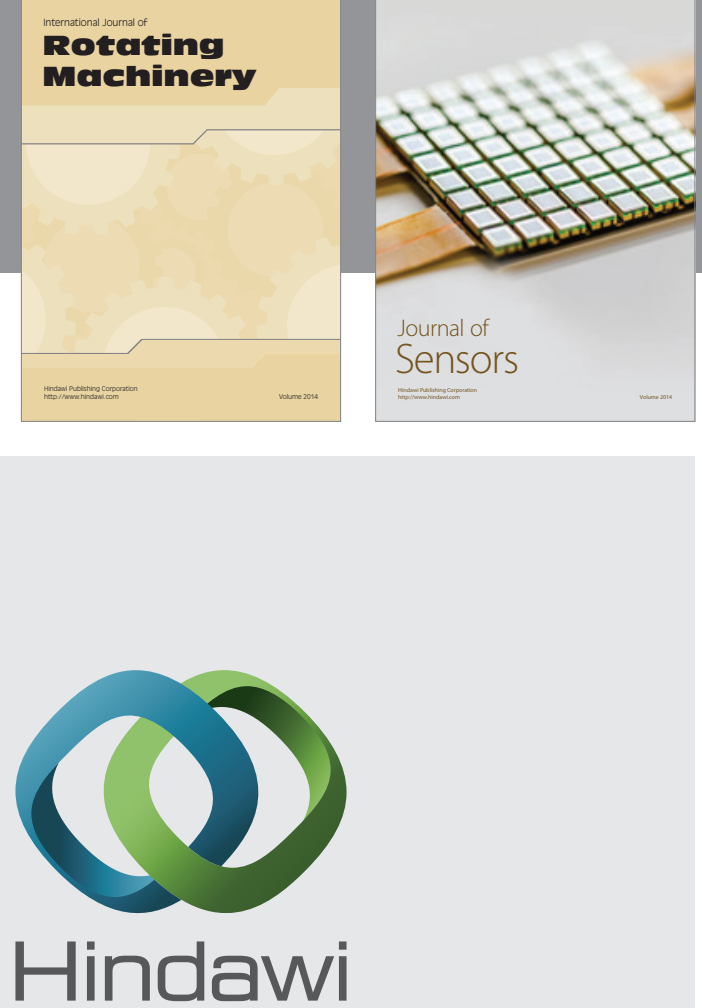

Submit your manuscripts at http://www.hindawi.com
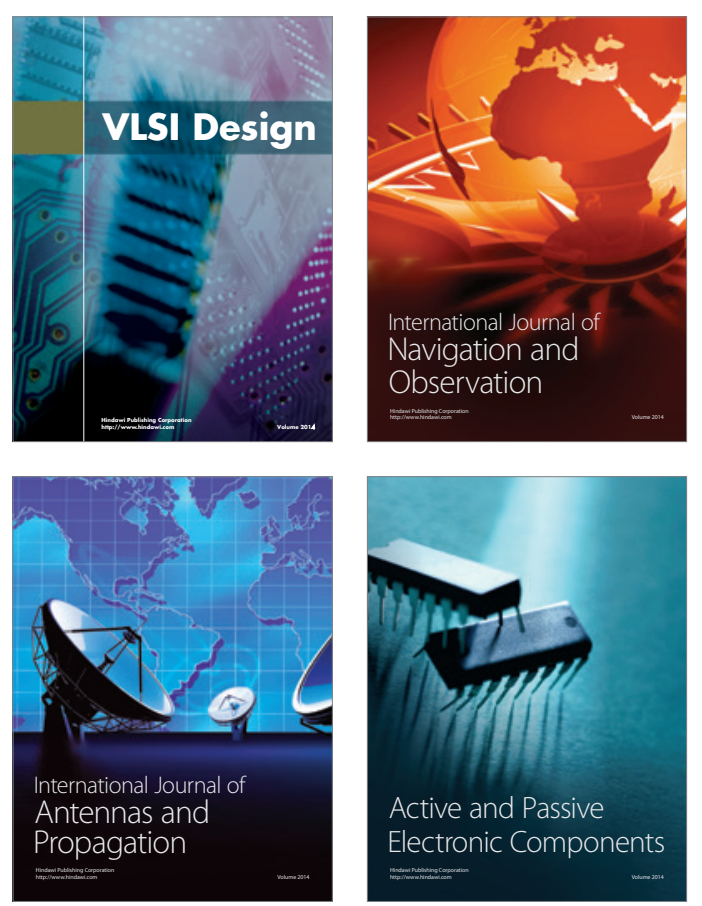
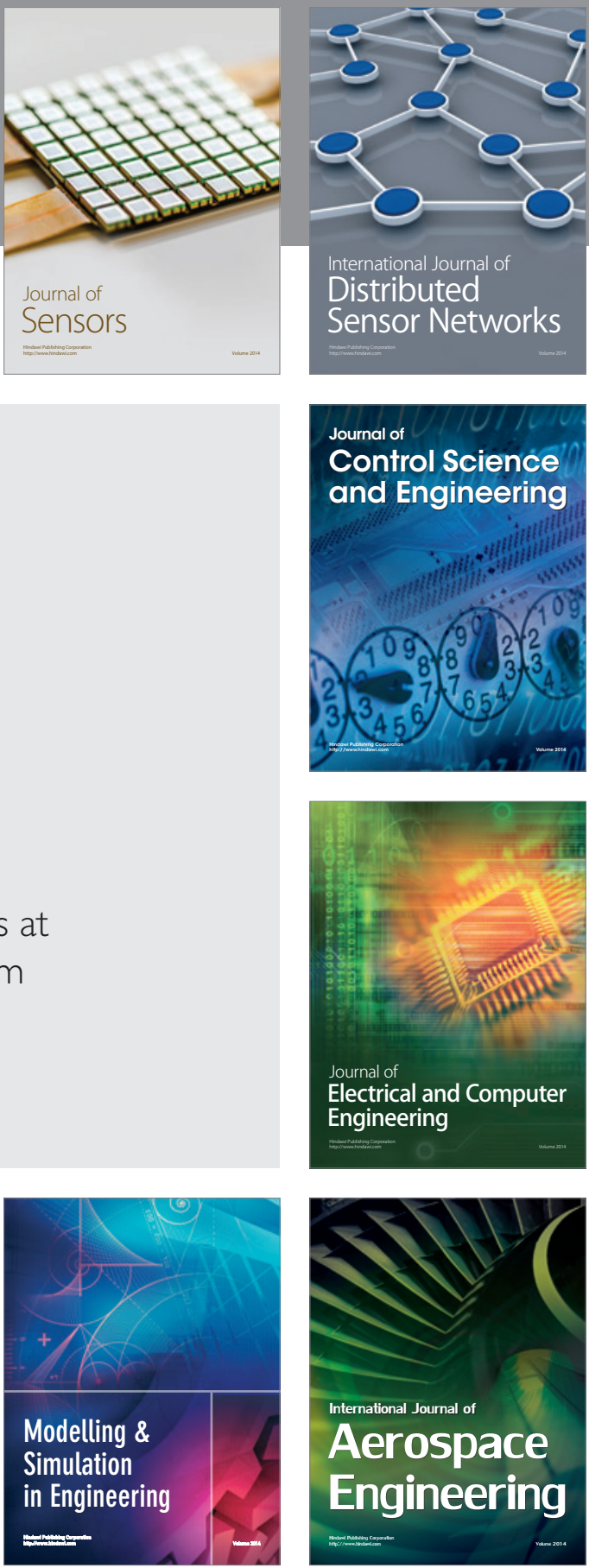

Journal of

Control Science

and Engineering
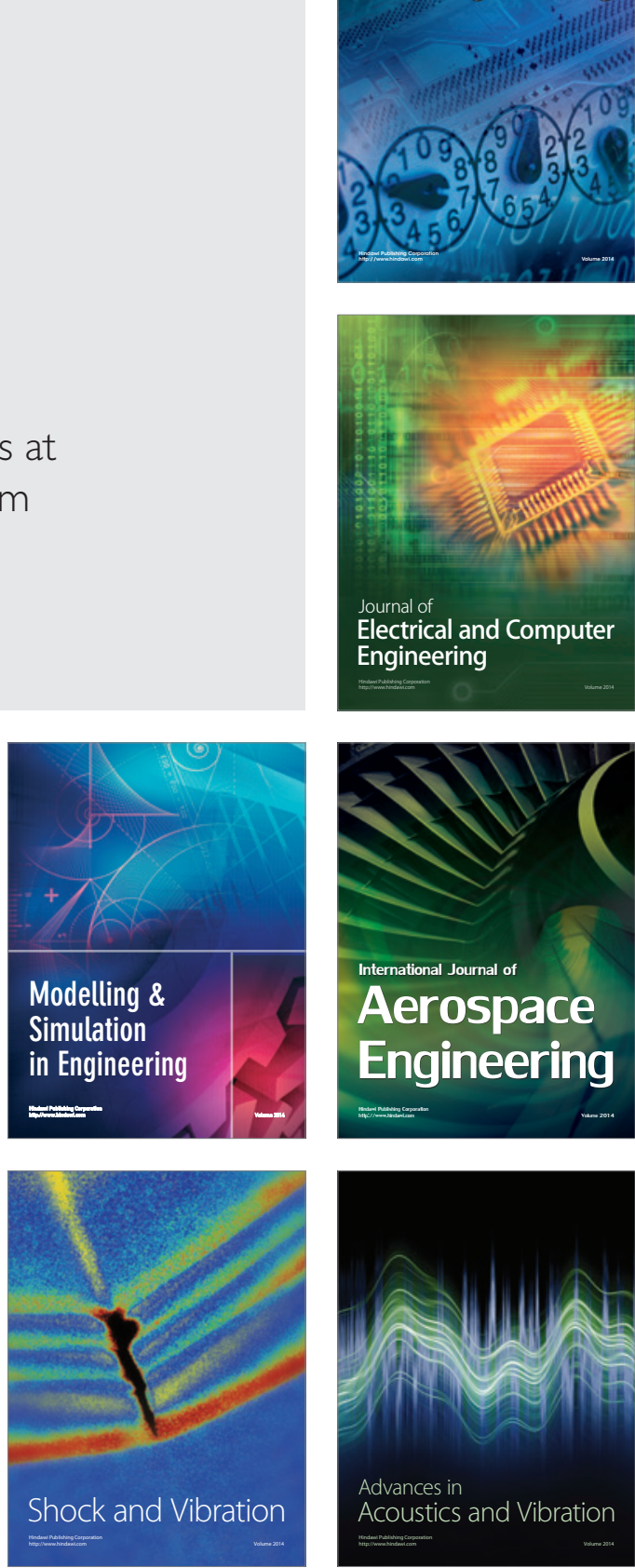